\title{
Smooth Scheduling for Electricity Distribution in the Smart Grid
}

\author{
Yingsong Huang, Student Member, IEEE, Shiwen Mao, Senior Member, IEEE, and R. M. Nelms, Fellow, IEEE
}

\begin{abstract}
The emergence of smart grid (SG) brings about many fundamental changes in electric power systems. In this paper, we study the problem of smooth electric power scheduling in power distribution networks. We introduce an electricity supply/demand model that takes into account the time-varying demands and their deadlines. We formulate a constrained nonlinear programming problem and incorporate the theory of majorization to develop algorithms that can compute smoothness optimal schedules for the deferrable load dominant system. An effective heuristic algorithm is also presented by extending the majorization-based algorithm for the general scenario with both priority loads and deferrable loads. After obtaining the smooth power schedule, a distributed user benefit maximization load control scheme is used to allocate the scheduled power to individual users, while maximizing their levels of satisfaction. The analysis and simulation results demonstrate the efficacy of the proposed algorithms on smooth electric power scheduling, peak power minimization, and reducing power generation cost.
\end{abstract}

Index Terms-Convex optimization, demand response (DR), majorization, power distribution, smart grid (SG).

\section{INTRODUCTION}

$\mathbf{T}$ HE emergence of smart grid (SG) brings about many fundamental changes in electric power systems [2]. Various new power electronics and information techniques are greatly advancing the control and management of energy and resources in the power system. For example, solid-state transformers can respond to signals from a facility or a household to change the voltage and other electric characteristics. On the user side, smart meters and smart facilities pervasively empower monitoring and controlling at all levels of power usage in response to power supply and market price fluctuations [2]. The twoway flows of electricity and information in SG are instrumental to the control and optimization of energy and resource allocation in the grid to achieve efficient, green, and robust energy systems.

Manuscript received March 30, 2013; revised March 10, 2014 and June 30, 2014; accepted July 9, 2014. Date of publication August 13, 2014; date of current version June 12, 2015. This work was supported in part by the National Science Foundation under Grant CNS-0953513 and through the Broadband Wireless Access and Applications Center site at Auburn University. This work was presented in part at IEEE GLOBECOM 2012-Workshop on Smart Grid Communications: Design for Performance. Part of this work was done when Y. Huang was pursuing the Ph.D. degree in electrical and computer engineering at Auburn University.

Y. Huang is with NetApp Inc., Pittsburgh, PA 15238 USA (e-mail: yzh0002@auburn.edu).

S. Mao and R. M. Nelms are with the Electrical and Computer Engineering Department, Auburn University, Auburn, AL 36849 USA (e-mail: smao@iee. org;nelmsrm@auburn.edu).

Digital Object Identifier 10.1109/JSYST.2014.2340231
In the SG paradigm, the next-generation distribution networks are capable of allowing users to control their loads in response to the dynamics in the grid. Demand response (DR) is an important technique to balance the power generation and demand in the grid [3]. One of the important goals of DR is to reduce the peak demand and smooth the power profile by scheduling user requests [2], [4]-[6]. With the twoway information flow among provider, users, and the market, various DR schemes based on real-time pricing and day-ahead load response concepts have been recently investigated [7][14]. Most of the existing DR schemes aim to maximize the social welfare or minimize the electricity payment under given demand requirements. Although revealing the intrinsic connection between pricing policies and DR, the problem of smooth electric power scheduling has not been explicitly addressed, which is the key issue in DR. It is shown that the simple offpeak pricing scheme may not be effective in mitigating the demand peak problem, because simply shifting the off-peak period may generate a new rebound peak [15].

In this paper, we address the challenging problem of smooth electric power scheduling in power distribution networks. A smooth electric power profile is very important and highly preferable in the power delivery system, which reduces plant operation cost and finally leads to the maximization of the social welfare. In addition, a smooth schedule also brings about the extra benefit of reducing the overall capital investment, which is hard to achieve through social welfare maximization. The power distribution system is typically organized as radial networks or interconnected networks. In this paper, we adopt a network structure as shown in Fig. 1 [16]. We assume that there is no randomness on the supply side. The supply may be conventional bulk generation, virtual power plant [17], which clusters many distributed renewable energy resources (DRERS) with a total capacity comparable with a traditional power plant, or a combination of these supply methods. Power generation is scheduled by a control center, which adopts a load-following generation strategy for the power network.

We assume that end users are equipped with smart meters and are capable of communicating with the distribution substation and the distribution control center (DCC) through a communication network and receiving commands from the DCC to adjust the user's electric energy consumption level [18]. The DCC schedules electricity supply on a daily basis, which is further divided into multiple time slots. The electricity usage requests at each user are classified into two categories: 1) priority load, which must be satisfied in every time slot, and 2) deferrable load, which should be satisfied before specific deadlines. Users classify the load for each type according to 


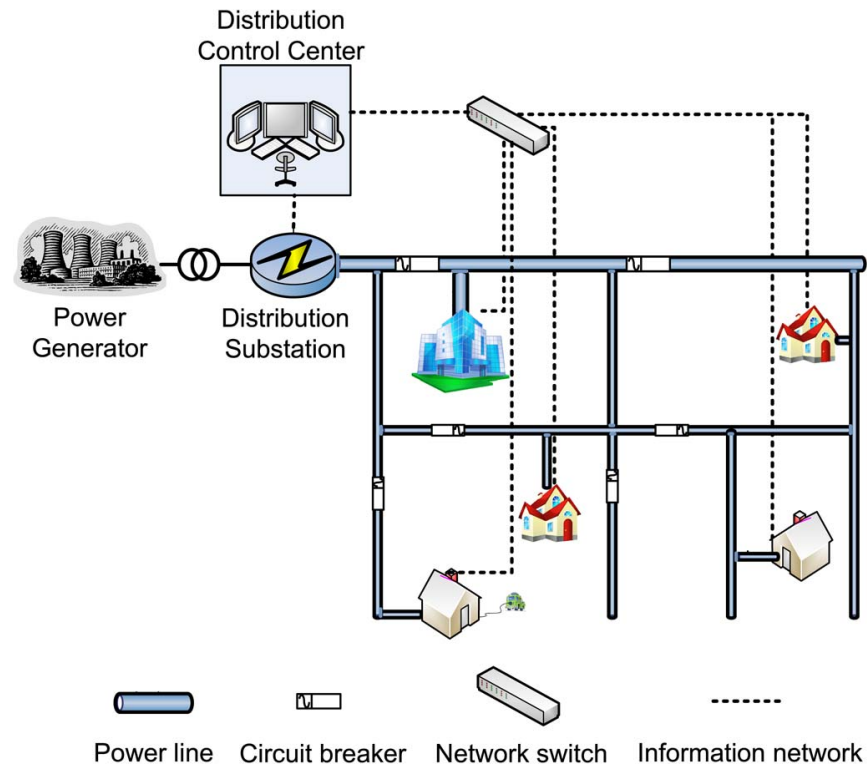

Fig. 1. Illustration of the electricity distribution network.

their preference [e.g., lighting, entertainment, or charging a plug-in hybrid electric vehicle (PHEV)] [3]. The DCC aggregates the demand profiles from the users through the aggregator [19] and smooths the aggregated electric power supply under the priority load and deferrable load deadline constraints.

The objective of the smooth electric power scheduling problem is to minimize the power variation during a daily period, based on the concept of day-ahead load response. A deterministic electricity supply/demand model is introduced with cumulative electricity demand/supply curves, which characterize the demand/supply relationship during the day. We find that the formulated problem suits well with the majorization theory, which concerns with the comparison and ordering of vectors with respect to the distribution of their elements [20]. Majorization has been used in solving optimization problems in the communications and networking area [21], [22]. In this paper, we present a majorization-based framework to develop two smooth electric power scheduling algorithms with low computational complexity. Once the smooth electric power profile for the entire network is obtained, a user benefit maximization load control algorithm will be executed to allocate the total amount of supply to the individual users, while maximizing their satisfaction of electricity usage. The proposed algorithms can achieve the minimum peak power, thus requiring smaller capacity for the generators, transmission lines, and transformers to support the same demand. Since electrical generation and transmission systems are generally designed to accommodate peak electric power [2], the smooth electric power schedule also helps to optimize the deployment and operation cost of the grid.

This paper extends an earlier five-page conference version [1] with the following new contributions: more detailed discussions, introduction of the preliminaries, an in-depth performance analysis of the proposed schemes, detailed proofs of the propositions and lemmas, and a comprehensive simulation evaluation of the proposed schemes. The remainder of this paper is organized as follows. We first present the system model and problem statement in Section II. We next provide a brief
TABLE I

NOTATION

\begin{tabular}{l|l}
\hline Symbol & Description \\
\hline $\mathbb{U}$ & $\begin{array}{l}\text { set of the electricity consumption users in the power } \\
\text { distribution networks }\end{array}$ \\
$N$ & total number of local electricity consumption user \\
$L$ & total number of slots \\
$\tau$ & slot period \\
$p_{n}(t)$ & $\begin{array}{l}\text { power consumption of user } n \text { in slot } t \\
\text { priority load electric energy of user } n \text { in slot } t\end{array}$ \\
$e_{n, p}(t)$ & deferrable load electric energy need to be fulfilled \\
$e_{n}, d(t)$ & since last deadline of user $n$ in slot $t$
\end{tabular}

introduction of majorization in Section III. The smooth electric power scheduling algorithms are described in Section IV, and their performance is evaluated in Section V. Related work is discussed in Section VI, and Section VII concludes this paper. The notation is summarized in Table I.

\section{Problem Statement}

\section{A. Load Demand Profile}

We consider a power distribution network with two-way flows of electricity and information. We assume $N$ users in the power distribution network, which may generate residential, commercial, and industrial load. Let $\mathbb{U}=\{1,2, \ldots, N\}$ be the set of users. The electric demand of a user is daily based. Without loss of generality, we assume that the one-day period is divided into $L$ time slots, each with length $\tau$. Let $p_{n}(t)$ be the power consumption of user $n$ in time slot $t$, which is time varying but remains constant within the time slot. Each user $n$ knows its own total daily demand, i.e., $E_{n}=\sum_{t=1}^{L} p_{n}(t) \tau$, and wishes to schedule the demand over the one-day period [12].

We assume that the total demand $E_{n}$ consists of two parts: the priority load and the deferrable load. The priority load should be strictly guaranteed in a time slot (e.g., for lighting), whereas the deferrable load can be flexibly served but with a specific deadline (e.g., charging a household battery or PHEVs). We define $e_{n, p}(t)$ and $e_{n, d}(t)$ as the electric energy for priority load in time slot $t$ and the deferrable load that must be satisfied by time slot $t$, respectively. The minimum demand 


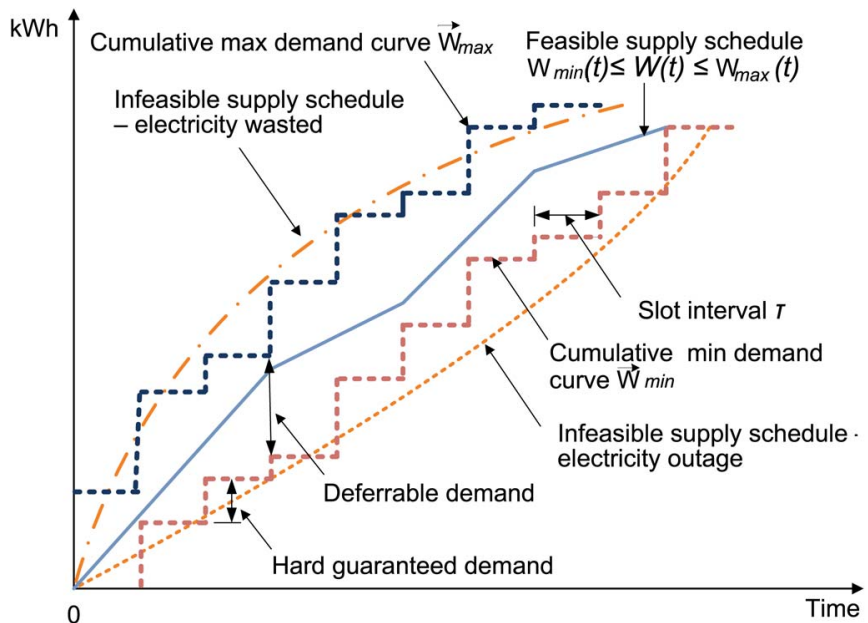

Fig. 2. Cumulative demand and supply curves.

of user $n$ in time slot $t$, which is denoted by $e_{n}^{\min }(t)$, is the sum of $e_{n, p}(t)$ and $e_{n, d}(t)$. Finally, let $e_{n}^{\max }(t)$ be the maximum possible demand for user $n$, which is limited by the amount of deferrable loads that have not been satisfied yet and the capacity of protective relays and switches of the users.

\section{B. Cumulative Demand and Supply Curves}

At the beginning of a day, the DCC will aggregate the individual demand profiles received by communicating with the smart meters and smart facilities via the communications network [18]. Let the total minimum electricity demand in time slot $t$ be $E_{\min }(t)=\sum_{n \in \mathbb{U}} e_{n}^{\min }(t)$. We have $E_{\min }(L)=$ $\sum_{n \in \mathbb{U}} E_{n}=\Phi$, since the daily aggregated demand of all users, which is denoted by $\Phi$, should finally be satisfied by the end of the day. We define the cumulative minimum demand curve $\vec{W}_{\min }$ as

$$
W_{\min }(t)=\sum_{l=1}^{t} E_{\min }(l), \quad 1 \leq t \leq L .
$$

We define the cumulative maximum demand curve $\vec{W}_{\max }$ to represent the maximum amount of electricity demand that can be consumed up to $t$ as $W_{\max }(t)=\min \left\{W_{\min }(t-\right.$ $\left.1)+\sum_{n \in \mathbb{U}}\left[e_{n}^{\max }(t)+\Delta e_{n}(t-1)\right], \Phi\right\}, \quad 1 \leq t \leq L$, where $\Delta e_{n}(t)=e_{n}^{\max }(t)-e_{n}^{\min }(t)$ is the deferrable load that can be served in slot $t$ but with deadlines later than $t$. To incorporate the priority load in the model, $W_{\max }(t)$ also satisfies $W_{\max }(t) \geq W_{\max }(t-1)+\sum_{n \in \mathcal{U}} e_{n, p}(t)$.

For given demand curves $\vec{W}_{\min }$ and $\vec{W}_{\max }$, we aim to find a feasible electricity schedule $\vec{W}$, which is the cumulative supply of electricity to the users that satisfies constraints $W_{\min }(t) \leq$ $W(t) \leq W_{\max }(t)$, for all $1 \leq t \leq L$, and $W(L)=\Phi$ (i.e., the total demand should be satisfied by the end of the day). The three cumulative curves are illustrated in Fig. 2, which are all nondecreasing over time.

The proposed demand and supply model is quite general. It does not assume any mathematical model for either the supply or the demand. It is more practical than the complex statistical models for supply and demand used in the literature
[2]. The cumulative curves represent the demand/supply status in the power distribution network. In each time slot $t, W_{\min }(t)$ tracks the priority load and the deferrable load with deadline $t$, whereas $W_{\max }(t)$ represents an upper bound of the possible consumption by time $t$. The gap between $W_{\max }(t)$ and $W_{\min }(t)$ may accommodate the future uncertainty of the electric power usage. The slope of $W(t)$, which is denoted by $P(t)$, corresponds to the scheduled electric power. The DCC aims to find an optimal schedule $W(t)$ for every time slot $t$ to achieve a specific control target. A feasible power supply schedule $\vec{P}=$ $[P(1), P(2), \ldots, P(L)]$ ensures that $\vec{W}$ lies between $\vec{W}_{\min }$ and $\vec{W}_{\text {max }}$ for all the $L$ time slots, thus preventing both outage events and energy waste.

It is shown in Fig. 2 that the feasible electric power schedule may not be unique. Among various feasible schedules, we are interested in the one that distributes electricity most smoothly among the $L$ time slots, i.e., the smoothness optimal schedule. Once the DCC obtains the smoothness optimal schedule, it can announce the schedule to the smart meters and smart utilities at the users' premises via the communication network, and the users can shape their demand to match the schedule (assuming cooperative users). Therefore, we can achieve smooth electricity generation, transmission, and consumption, which is highly preferable for the grid design and operation [2].

\section{Smooth Power Scheduling Problem}

Based on the demand and supply model, we formulate the smooth power scheduling problem here. Let $\bar{P}=\Phi /(L \tau)$ be the average power consumption in the power distribution network through the daily period. The scheduled power for each time slot is $P(t)=(W(t)-W(t-1)) / \tau=E(t) / \tau$. The smoothness optimal schedule minimizes the variations of the supplied power over the entire period, i.e.,

$$
\begin{array}{ll}
\operatorname{maximize}: & \mathcal{S}(\vec{P}) \\
\text { subject to : } & W_{\min }(t) \leq W(t) \leq W_{\max }(t), \text { for all } t \\
& W(L)=W_{\min }(L)=W_{\max }(L)=\Phi \\
& P(t)=[W(t)-W(t-1)] \tau \\
& \geq \frac{E_{p}(t)}{\tau}, \text { for all } t
\end{array}
$$

where $\mathcal{S}(\vec{P})$ is the smoothness of a schedule $\vec{P}$, and $E_{p}(t)=$ $\sum_{n \in \mathbb{U}} e_{n, p}(t)$ is the total priority load in time slot $t$.

Generally, smoothness can be measured by different metrics, such as variance and cumulative absolute difference. Each smoothness measure leads to a different objective function in problem (2), whereas the solution to the problem will then depend on the specific form of the objective function. In addition, the smoothness measures are generally nonlinear, making the problem nontrivial to solve. In this paper, we resort to the mathematical theory of majorization [20], which explicitly addresses the unique mathematical notion for smoothness. Applying majorization theory, we will see that, for an arbitrary smoothness objective function in problem (2) that satisfies the Schur-convex properties [20], the problem can be solved by 
a universal algorithm in polynomial time. For brevity in the deduction, we minimize the load variance in the rest of this paper, whereas the solution algorithms developed in Section IV apply to any objective function that is Schur-convex.

We first consider the case where the deferrable load is the dominant component [23], i.e., $E_{p}(t) \approx 0$. Problem (2) is then reduced to problem (3), i.e.,

$$
\begin{aligned}
\text { minimize : } & \sum_{t=1}^{L}[P(t)-\bar{P}]^{2} / L \\
\text { subject to : } & W_{\min }(t) \leq W(t) \leq W_{\max }(t), \text { for all } t \\
& W(L)=\Phi \\
& P(t)=\frac{[W(t)-W(t-1)]}{\tau}, \text { for all } t .
\end{aligned}
$$

This problem fits well with the majorization theory, since the objective function is Schur-convex [20]. We briefly review majorization preliminaries in Section III. Applying majorization, we will design a smooth electric power scheduling algorithm for solving problem (3) in Section IV-A. We will then extend the algorithm for solving problem (2) in Section IV-C.

\section{MAJORIZATION PRELIMINARIES}

The majorization theory [20] describes the "less spreading out" or the "more nearly equal" properties of the elements of a vector comparing with the elements of another vector. It concerns with the problem of ordering vectors with nonnegative real elements, as well as order-preserving functions. For simplicity, all the vectors here are row vectors.

Definition 1: For two n-element vectors $\vec{X}=$ $\left(x_{1}, x_{2}, \ldots, x_{n}\right)$ and $\vec{Y}=\left(y_{1}, y_{2}, \ldots, y_{n}\right)$, with elements sorted in the nonincreasing order as $x_{1} \geq x_{2} \geq \cdots \geq x_{n} \geq 0$ and $y_{1} \geq y_{2} \geq \cdots \geq y_{n} \geq 0 . \vec{X}$ is said to be majorized by $\vec{Y}$, which is denoted by $\vec{X} \prec \vec{Y}$, if 1) $\sum_{i=1}^{t} x_{i} \leq \sum_{i=1}^{t} y_{i}$, $t=1,2, \ldots, n-1$, and 2) $\sum_{i=1}^{n} x_{i}=\sum_{i=1}^{n} y_{i}$ [20].

Definition 2: A real-valued function $\phi$ defined on a set $\mathcal{A} \subset$ $\mathcal{R}^{n}$ is said to be Schur-convex on $\mathcal{A}$ if $\vec{X} \prec \vec{Y}$ on $\mathcal{A} \Rightarrow \phi(\vec{X}) \leq$ $\phi(\vec{Y})[20]$.

Schur-convex functions have the "order-preserving" property, which bridges majorization and optimization. Schurconvex functions can be validated with the following fact.

Fact 1: If $\phi$ is symmetric and convex, then $\phi$ is Schurconvex. Consequently, $\vec{X} \prec \vec{Y}$ implies $\phi(\vec{X}) \leq \phi(\vec{Y})$ [20].

This fact provides connection between ordering and its orderpreserving functions. By this fact, we may solve the minimization problem by generating the most spreading out vector as the solution. If $\phi=\sum g$ and $g$ is continuous convex, then we have the following fact.

Fact 2: $\sum_{i} g\left(x_{i}\right) \leq \sum_{i} g\left(y_{i}\right) \Leftrightarrow \vec{X} \prec \vec{Y}$ holds for all continuous convex functions $g: \mathcal{R} \rightarrow \mathcal{R}$ [20].

Lemma 1: Let $\vec{X}=\left(\vec{X}_{1}, \ldots, \vec{X}_{K}\right)$ and $\vec{Y}=\left(\vec{Y}_{1}, \ldots, \vec{Y}_{K}\right)$, where each element has dimension $J_{i}$ and satisfying $\vec{X}_{i} \prec \vec{Y}_{i}$ for all $i$. Then, $\vec{X} \prec \vec{Y}$.

Proof: Let $g$ be the continuous convex function $\mathcal{R} \rightarrow \mathcal{R}$. By Fact 2, $\vec{X}_{i} \prec \vec{Y}_{i} \Leftrightarrow \sum_{j=1}^{J_{i}} g\left(x_{i}^{j}\right) \leq \sum_{j=1}^{J_{i}} g\left(y_{i}^{j}\right) \Rightarrow$ $\sum_{i=1}^{K} \sum_{j=1}^{J_{i}} g\left(x_{i}^{j}\right) \leq \sum_{i=1}^{K} \sum_{j=1}^{J_{i}} g\left(y_{i}^{j}\right) \Leftrightarrow \vec{X} \prec \vec{Y}$.

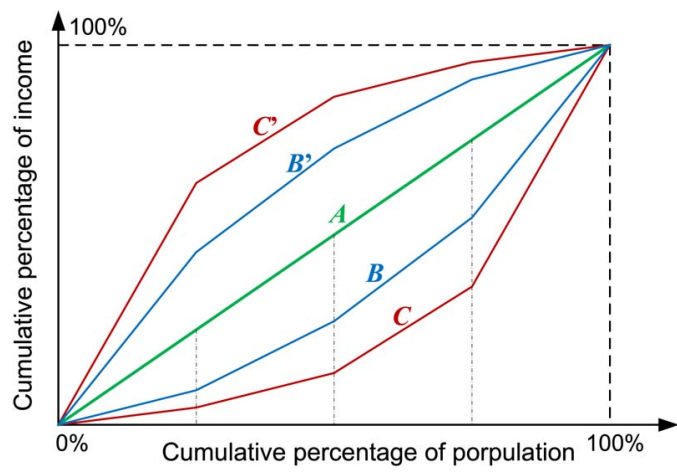

Fig. 3. Illustration of different Lorenz curves.

Let $\quad \vec{X}=(\bar{x}, \ldots, \bar{x}), \quad \vec{Y}=\left(y_{1}, \ldots, y_{n}\right), \quad$ and $\quad \vec{Z}=$ $\left(z_{1}, \ldots, z_{n}\right)$, where $\sum_{i=1}^{n} y_{i}=\sum_{i=1}^{n} z_{i}=n \bar{x}$. If the elements in each vector are nondecreasing, we can plot the normalized points of $\vec{X} /(n \bar{x}), \vec{Y} /(n \bar{x})$, and $\vec{Z} /(n \bar{x})$ as in Fig. 3. The elements of vectors can be interpreted as the incomes of individuals, as in a Lorenz curve, which evaluates the social income inequality [24]. The curves show the normalized cumulative proportion of the income versus the cumulative percentage of population. The normalized vector $\vec{X}$ forms a straight curve $A$, which corresponds to the equal distribution. Normalized vectors $\vec{Y}$ and $\vec{Z}$ represent unequal distributions and bent in the middle, which is denoted by curves $B$ and $C$ in the figure, respectively. We call them bow curves in the convex shape. The more the Lorenz curve bents, the higher the concentration of the elements; the bow curve closer to $A$ represents a more even distribution [20]. From the perspective of majorization, we have $\vec{X} \prec \vec{Y} \prec \vec{Z}$. Similarly, we can shuffle the elements in $\vec{X} /(n \bar{x})$ and $\vec{Y} /(n \bar{x})$ to get two new Lorenz curves $B^{\prime}$ and $C^{\prime}$. We call these bow curves in the concave shape. Since the order of the vectors plays no role in majorization, $\vec{X} \prec \vec{Y} \prec \vec{Z}$ still holds for concave curves.

Proposition 1: The objective function of problems (2) and (3) is Schur-convex.

Proof: The proof directly follows Fact 1, due to the symmetric and convex of the objective function in problems (2) and (3).

\section{Smooth Electric Power SCHEduling}

\section{A. SEPS-DL Algorithm}

We first develop a smooth electric power scheduling for deferrable load (SEPS-DL) algorithm based on majorization. With Proposition 1, we convert the optimization problem (3) into an ordering problem of vectors, each representing a feasible schedule. Thus, we solve problem (3) by computing the most evenly distributed electric power schedule that is feasible for the entire period. Obviously, the most evenly distributed schedule is $\vec{P}^{\text {opt }}=[\Phi /(L \tau), \ldots, \Phi /(L \tau)]$, corresponding to having the average power consumption $\bar{P}$ in each time slot. However, due to time-varying user demands, $\vec{P}^{\text {opt }}$ may not be feasible. In general, each feasible schedule is piecewise linear with a set of power changing points, where the scheduled power increases or decreases to prevent outage events or electric energy waste. 

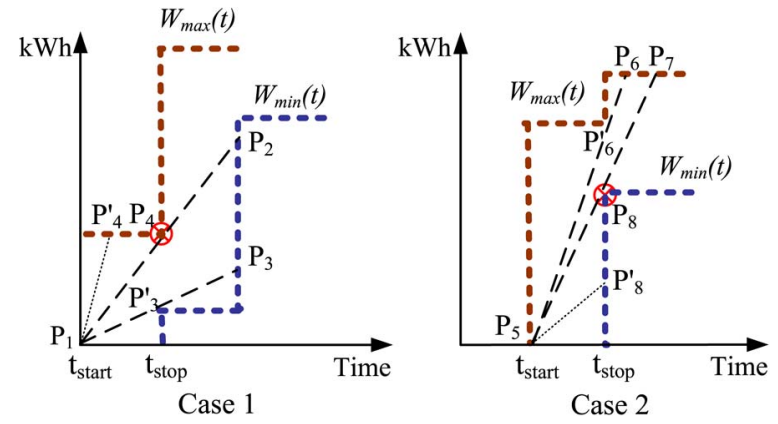

Fig. 4. Illustration of the operation of the SEPS-DL algorithm.

The proposed SEPS-DL algorithm generates a feasible piecewise linear schedule, while keeping each piece as long as possible into the future and the power variation as small as possible. The algorithm is illustrated with the examples in Fig. 4. Starting from $t_{\text {start }}$, SEPS-DL first computes two probe lines.

1) One probe line from $t_{\text {start }}$ to the next corner point of $W_{\max }(t)$, which can go the farthest into the future without causing outage events or energy waste (e.g., lines $\mathrm{P}_{1} \mathrm{P}_{4}$ in Case 1 and $\mathrm{P}_{5} \mathrm{P}_{6}^{\prime}$ in Case 2 in Fig. 4). The power of this probe line is $P_{\max }(t)=\left[W_{\max }(t)-\right.$ $\left.W\left(t_{\text {start }}\right)\right] /\left[t-t_{\text {start }}\right]$.

2) The other probe line from $t_{\text {start }}$ to the next corner point of $W_{\min }(t)$, which can go the farthest into the future without causing outage events or energy waste (e.g., lines $\mathrm{P}_{1} \mathrm{P}_{3}^{\prime}$ in Case 1 and $\mathrm{P}_{5} \mathrm{P}_{8}$ in Case 2). The power of this probe line is $P_{\min }(t)=\left[W_{\min }(t)-W\left(t_{\text {start }}\right)\right] /\left[t-t_{\text {start }}\right]$.

All feasible schedules should reside between the two probe lines in order to go farther into the future (i.e., to be smooth). Moreover, when the two probe lines are ended, they must hit both on either $W_{\max }(t)$ or both on $W_{\min }(t)$. Otherwise, we can always adjust one of the probe lines to make it go even farther into the future. For example, see lines $\mathrm{P}_{1} \mathrm{P}_{3}$ and $\mathrm{P}_{1} \mathrm{P}_{4}^{\prime}$ in Case 1 in Fig. 4. We can use line $\mathrm{P}_{1} \mathrm{P}_{2}$ (which goes farther into the future) to replace line $\mathrm{P}_{1} \mathrm{P}_{4}^{\prime}$, and both probe lines hit $W_{\min }(t)$ eventually (also see lines $\mathrm{P}_{5} \mathrm{P}_{6}$ and $\mathrm{P}_{5} \mathrm{P}_{8}^{\prime}$ in Case 2). In Case 2 in the figure, both probe lines $\mathrm{P}_{5} \mathrm{P}_{6}$ and $\mathrm{P}_{5} \mathrm{P}_{7}$ hit $W_{\max }(t)$.

If both probe lines hit $W_{\min }(t)$ (i.e., Case 1 in Fig. 4), any feasible schedule for this interval will also hit $W_{\min }(t)$, since it must lie between the two probe lines. We then trace back the upper probe line (i.e., line $\mathrm{P}_{1} \mathrm{P}_{2}$ ) to find the latest time when the schedule just satisfies the maximum demand (i.e., point $\mathrm{P}_{4}$ at time $t_{\text {stop }}$ ). Then, segment $\mathrm{P}_{1} \mathrm{P}_{4}$ will be chosen as the schedule for the interval $\left[t_{\text {start }}, t_{\text {stop }}\right)$, with power $\left(W_{\text {max }}\left(t_{\text {stop }}\right)-W\left(t_{\text {start }}\right)\right) /\left(t_{\text {stop }}-t_{\text {start }}\right)$.

If both probe lines hit $W_{\max }(t)$ (i.e., Case 2 in Fig. 4), any feasible schedule for this interval will also hit $W_{\max }(t)$. We then trace back the lower probe line (i.e., line $\mathrm{P}_{5} \mathrm{P}_{7}$ ) to find the latest time when the schedule just satisfies the minimum demand (i.e., point $\mathrm{P}_{8}$ at time $t_{\text {stop }}$ ). Then, segment $\mathrm{P}_{5} \mathrm{P}_{8}$ will be chosen as the schedule for the interval $\left[t_{\text {start }}, t_{\text {stop }}\right)$, with power $\left(W_{\text {min }}\left(t_{\text {stop }}\right)-W\left(t_{\text {start }}\right)\right) /\left(t_{\text {stop }}-t_{\text {start }}\right)$.

After the schedule for $\left[t_{\text {start }}, t_{\text {stop }}\right)$ is determined, we set $t_{\text {start }}=t_{\text {stop }}$ and repeat the preceding procedure to find the schedule for the next time interval.
As shown in Algorithm 1, SEPS-DL probes for the longest feasible power starting from $t_{\text {start }}$ in Steps 4-8. In Steps $9-12$, the power for the interval $\left[t_{\text {start }}, t_{\text {stop }}\right)$ is determined depending on which of the two cases it is, as illustrated in Fig. 4. Steps 14-15 are for the case that the power does not change in the time slot. Step 16 resets the variables to start the computation for the next segment of $P(t)$.

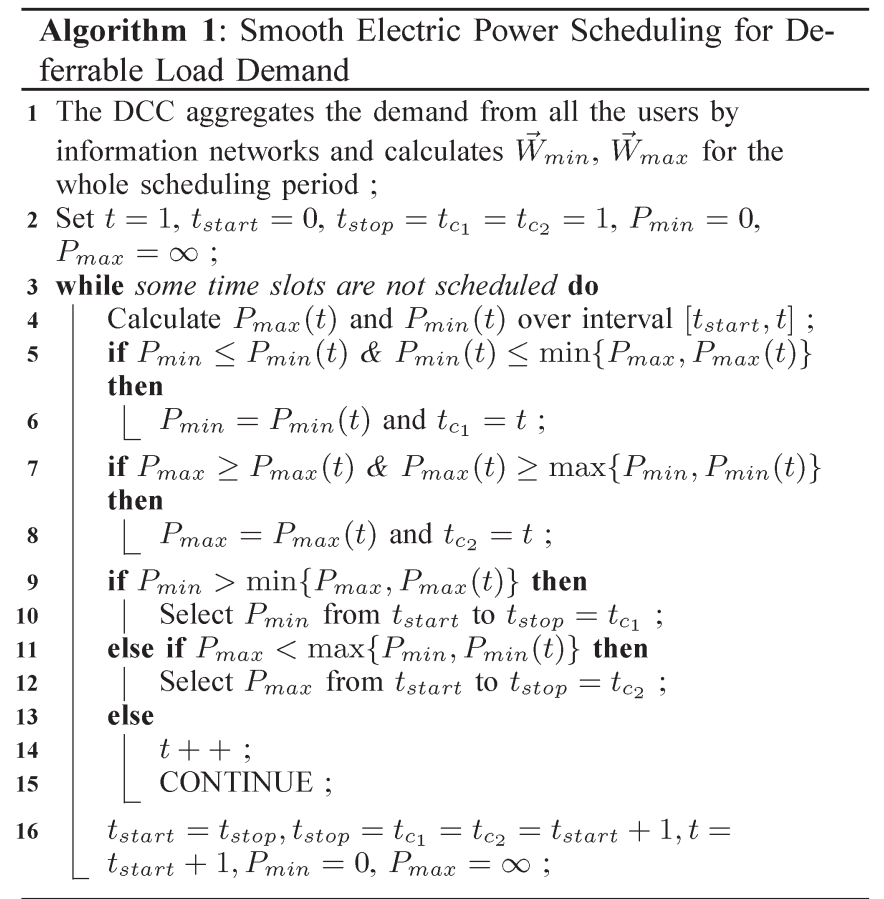

\section{B. Performance of SEPS-DL}

The SEPS-DL algorithm is easy to implement. It can be shown that SEPS-DL has the following properties.

Proposition 2: SEPS-DL is smoothness optimal for problem (3).

Proof: The proof is similar to that in [21]. The schedule computed by SEPS-DL, i.e., $\vec{P}^{*}$, could be a straight line or, in the general case, consists of one or more convex and concave segments. If $\vec{P}^{*}$ is a straight line, it is obvious that $\vec{P}^{*} \prec \vec{P}_{k}$ for any other $\vec{P}_{k}$, and it is smooth optimal. In the general case, we need to show $\vec{P}^{*} \prec \vec{P}_{k}$, for all $k$ in every convex or concave segment. Then, according to Lemma 1 , we have $\vec{P}^{*} \prec \vec{P}_{k}$ for all $k$, and it is optimal.

Let $\vec{P}_{k}$ denote an arbitrary feasible schedule. We introduce an auxiliary schedule $\vec{P}_{1}$, which intersects with $\vec{P}^{*}$ at all its power changing points in every convex segment and with $\vec{P}_{k}$ at all its power changing points in every concave segment, as shown in Fig. 5.

First, we show that $\vec{P}^{*} \prec \vec{P}_{1}$. Consider a convex segment of $\vec{P}^{*}$. Because $\vec{P}_{1}$ intersects with $\vec{P}^{*}$ at all the power changing points of $\vec{P}^{*}$, we have $\vec{P}^{*}=\vec{P}_{1}$ in all the convex segments. For a concave segment of $\vec{P}^{*}$, the endpoints of the concave segment should be the last (first) power changing point of the previous (next) convex segment, where $\vec{P}^{*}$ intersects with $\vec{P}_{1}$. The power changing points within the concave segment are all on $W_{\min }(t)$, as in SEPS-DL. Therefore, $\vec{P}_{1}$ is an outer concave 


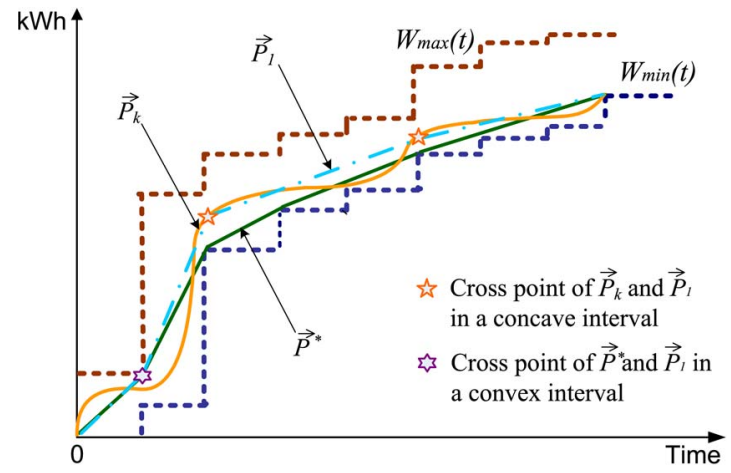

Fig. 5. Illustration for the optimality proof of the SEPS-DL algorithm.

curve above $\vec{P}^{*}$ in this segment. We have $\vec{P}^{*} \prec \vec{P}_{1}$ for all the concave segments. It follows that $\vec{P}^{*} \prec \vec{P}_{1}$ according to Lemma 1.

We next prove that $\vec{P}_{1} \prec \vec{P}_{k}$. For a convex segment of $\vec{P}^{*}$, the endpoints of the convex segment should be the last (first) power changing point of the previous (next) concave segment, where $\vec{P}_{k}$ intersects with $\vec{P}_{1}$. The power changing points of $\vec{P}_{1}$ (or, of $\vec{P}^{*}$ ) in the convex segment are all on $W_{\max }(t)$. Therefore, $\vec{P}_{k}$ is an outer convex curve below $\vec{P}_{1}$ in this segment. It follows that $\vec{P}_{1} \prec \vec{P}_{k}$ in all the convex segments. In a concave segment, we have either $\vec{P}_{k}=\vec{P}_{1}$ or $\vec{P}_{k} \prec \vec{P}_{1}$, because $\vec{P}_{1}$ intersects with $\vec{P}_{k}$ at each power changing point. Thus, we obtain $\vec{P}_{1} \prec \vec{P}_{k}$ for all the concave segments, and $\vec{P}_{1} \prec \vec{P}_{k}$ according to Lemma 1 .

Finally, we have $\vec{P}^{*} \prec \vec{P}_{1} \prec \vec{P}_{k}$. Proposition 1 states that problem (3) is Schur-convex and order preserving. It follows from Fact 1 that $\vec{P}^{*}$ is optimal to problem (3).

Proposition 3: The optimal power schedule computed by SEPS-DL is unique.

Proof: Suppose $\vec{P}^{*}$ is not unique. Then, there exists $\vec{P}^{\prime} \prec$ $\vec{P}_{k}$, for all $k$, and $\vec{P}^{\prime} \neq \vec{P}^{*}$. $\vec{P}^{\prime}$ must have a different set of power changing points than that of $\vec{P}^{*}$. According to the proof of Proposition 2, we can construct an auxiliary schedule $\vec{P}_{1}$, such that $\vec{P}^{*} \prec \vec{P}_{1} \prec \vec{P}^{\prime}$ holds true, which contradicts the assumption that $\vec{P}^{\prime}$ is optimal.

Proposition 4: The complexity of SEPS-DL is $\mathcal{O}\left(L^{2}\right)$.

Proof: In the worst case, SEPS-DL computes the optimal schedule for each time slot by probing the full length of the remaining time slots (as in Steps 4-10 in Algorithm 1). The worst case complexity is $\sum_{i=L}^{1} i=L(L+1) / 2 \Rightarrow \mathcal{O}\left(L^{2}\right)$.

Proposition 5: The smooth electric power schedule computed by SEPS-DL has the smallest peak power.

Proof: From Proposition 2, we have $\vec{P}^{*} \prec \vec{P}_{k}$, for all $k$. We can sort the elements in both $\vec{P}^{*}$ and $\vec{P}_{k}$ in the nonincreasing order. According to the definition of majorization in Definition 1 , the first element in the reordered $\vec{P}^{*}$ is not greater than that of $\vec{P}_{k}$, which means that the largest element in $\vec{P}^{*}$ is not greater than that of $\vec{P}_{k}$. Thus, the schedule generated by SEPS-DL has the lowest peak power.

Proposition 5 is highly desirable for grid design and operation. A lower peak power allows the operator to deploy generators, transformers, and power transmission lines with smaller capacity in the grid, thus reducing the capital investment. In addition, the grid can be alleviated of the power usage burden during peak hours.
Finally, we consider the power generation cost for the schedule computed by SEPS-DL. Without loss of generality, we assume that the fuel cost at time slot $t$ is $C(t)=g(P(t), \theta(t))$, which is a nondecreasing convex function of the supplied power $P(t)$ [13]. This assumption is generally practical, e.g., the fuel cost for the electric energy generation is usually considered as a quadratic function of its power generation [25]. We also assume that the cost $C(t)$ is affected by a random factor $\theta(t)$, which corresponds to the cost uncertainty during the period, such as disturbances from the sources of fuel and market price. We assume that $\theta(t)$ is independent and identically distributed (i.i.d.) over the time slots. Thus, the minimization of the expectation of the total cost over the period of $L$ time slots is

$$
\begin{array}{ll}
\text { Minimize : } & \sum_{t=1}^{L} \mathbb{E}\{g(P(t), \vec{\theta}(t))\} \\
\text { subject to : } & W_{\min }(t) \leq W(t) \leq W_{\max }(t), \text { for all } t \\
& \sum_{t=1}^{L} W(t)=\Phi \\
& P(t)=\frac{[W(t)-W(t-1)]}{\tau}, \text { for all } t .
\end{array}
$$

Due to the convexity of the cost function $g(\cdot)$ with respect to $P(t)$, problem (4) is similar to problem (3), except for the random variable $\theta(t)$. We resort to stochastic majorization (rather than ordinary majorization) to solve this constrained nonlinear stochastic programming problem.

Lemma 2: The objective function of problem (4) is an increasing Schur-convex function.

Proof: The i.i.d. random variables $\theta(t)$ are exchangeable for all $t$. The objective function (4) can be rewritten as

$$
G(\vec{P})=\mathbb{E}\left\{\sum_{t=1}^{L} g(P(t), \theta(t))\right\}
$$

where $g(P(t), \theta(t))$ is convex and increasing with respect to $P(t)$ for each realization of $\theta(t)$, and $\sum_{t=1}^{L} g(P(t), \theta(t))$ is a symmetric, convex, and increasing function with respect to $P(t)$. According to [20, Proposition 11.B.5], the expectation $G(\vec{P})$ is symmetric, convex, and increasing. Following Fact 1 , the objective function (4) is Schur-convex and increasing.

Proposition 6: SEPS-DL is generation cost optimal.

Proof: According to Lemma 2, the solution of problem (4) is equivalent to finding the optimal power vector $\vec{P}^{*}$, which is majorized by any other feasible power vectors. Thus, the smooth optimal solution $\vec{P}^{*}$ from Algorithm 1 is also the solution to problem (4). The proposed SEPS-DL achieves fuel cost optimal for power generation.

\section{Extension to the General Case}

We next extend SEPS-DL to solve the general case problem (2). With the priority load, a feasible power schedule should satisfy $P(t) \geq E_{p}(t) / \tau$ in every time slot. The presence of priority load enforces new constraints on the feasibility of the schedules. For example, consider the aggregated cumulative priority load curves and deferrable load curves shown in 


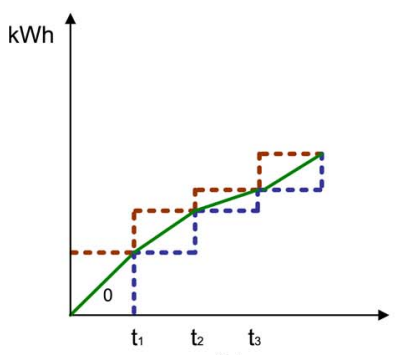

(a)

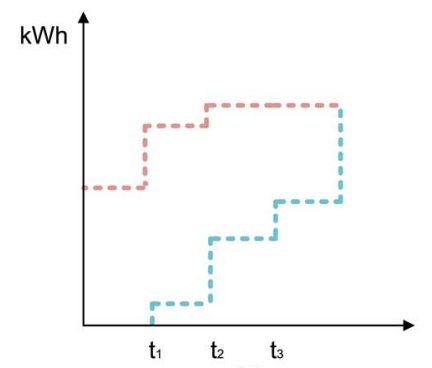

(b)

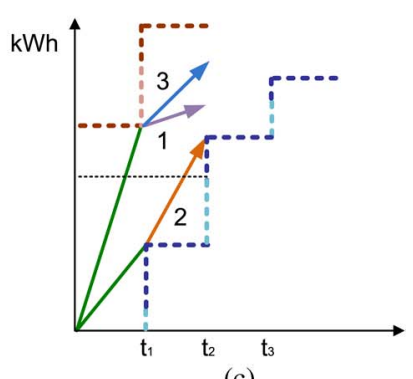

(c)

Fig. 6. Smooth power scheduling with priority load. Note that, although not deferrable, the cumulative priority load can be also represented with two curves, as shown in (a), where the maximum and minimum curves meet at each of the corners. (a) Cumulative priority load curves. (b) Cumulative deferrable load curves. (c) Aggregated cumulative load curves.

Fig. 6(a) and (b). According to the definition of feasible power supply schedule in SEPS-DL, both segments 1 and 2 in Fig. 6(c) are feasible. However, it can be seen that segment 1 actually cannot provide enough power to satisfy the priority load in time slot $t_{2}$, since its slope is smaller than the required slope [i.e., that of segment 0 in Fig. 6(a)].

To solve this problem, we develop the general smooth electric power scheduling algorithm (GSEPS), which is based on SEPS-DL. Recall that SEPS-DL assumes no priority load. During the execution, we first use SEPS-DL to compute a feasible power schedule segment. The generated power segment is then compared with the priority load. If the SEPS-DL generated power segment $P^{*}(t)$ is less than the priority load in time slot $t$, GSEPS will increase $P^{*}(t)$ to the priority load [e.g., see segment 3 in Fig. 6(c)]. Then, SEPS-DL will continue to compute further segments of the schedule by setting the new starting point to $\left(t, W^{*}(t)\right)$ until the entire schedule is computed.

Although the performance of SEPS-DL is optimal with respect to smoothness, peak reduction, and generation cost, GSEPS is a heuristic extension of SEPS-DL to handle the priority load. With the priority load, we cannot make the same optimality claims. In Section V, we use simulations to demonstrate that GSEPS still achieves very good performance with respect to both smoothness and cost effectiveness.

\section{Power Allocation Among Individual Users}

Once the smooth electric power schedule is obtained, the DCC announces the schedule to all the users and requests them to control their loads to match the supplied electric energy $W^{*}(t)-W^{*}(t-1)=P^{*}(t) \tau$ in each time slot $t$. To divide the total supply $W^{*}(t)$ among the $N$ users, we assume a benefit function $U_{n}\left(p_{n}(t)\right)$ for each user $n$, which is a nondecreasing concave function [13] and represents the level of satisfaction of the user when receiving $p_{n}(t)$ in time slot $t . U_{n}(t)$ may be different for different users or time varying for different time slots, reflecting the heterogeneous utility in the power network.

We assume that the users are assumed to be cooperative in the power allocation step, since their priority load will be guaranteed in this step [distributed user benefit maximization load control algorithm (DUBMLC)]. The goal of this step is to maximize the user utility. User utility function is a function of the user's power usage, e.g., a mapping from a users' usage amount to its electricity bill amount. The utility function can be varying from time slot to time slot (e.g., from high price in the on-peak period to low price in the off-peak period). We assume that the users are rational and are willing to participate since doing so will reduce their electricity bill. Therefore, there is incentive for the users to participate in the power allocation step. This method belongs to the real-time pricing strategy, and this approach is widely used in related works [14]. For brevity, we assume that the utility functions are not time varying in the following discussions. The extension to the timevarying case will not affect the analysis and conclusion, if the utility functions all satisfy the previous nondecreasing concave property.

We then develop an algorithm that maximizes the sum of the benefit functions of all users in the power distribution network. The maximization of the total benefit under the smooth schedule constraint can be formulated in each time slot $t$ as follows:

$$
\begin{array}{ll}
\text { maximize : } & \sum_{n \in \mathbb{U}} U_{n}\left(p_{n}(t)\right) \\
\text { subject to : } & p_{n}^{\min }(t) \leq p_{n}(t) \leq p_{n}^{\max }(t), \text { for all } n \\
& \sum_{n \in \mathbb{U}} p_{n}(t)=\frac{\left[W^{*}(t)-W^{*}(t-1)\right]}{\tau}
\end{array}
$$

where $p_{n}^{\max }(t)$ and $p_{n}^{\min }(t)$ are the maximum and minimum power consumptions of user $n$ in slot $t$, respectively.

Problem (5) is a convex optimization problem, which can be solved effectively with a convex optimization solver. In case that the DCC may not know the exact parameters of individual utility functions in practice, we develop a DUBMLC based on dual decomposition [26] to solve problem (5). For brevity, we omit the time slot notation $t$ in the following equations.

First, we introduce the nonnegative Lagrange multiplier $\nu$ and derive the Lagrange function, as follows:

$$
\begin{aligned}
\mathcal{L}\left(p_{n}, \nu\right) & =\sum_{n \in \mathbb{U}} U_{n}\left(p_{n}\right)+\nu\left(W-\sum_{n \in \mathbb{U}} p_{n}\right) \\
& =\sum_{n \in \mathbb{U}} \mathcal{L}_{n}\left(p_{n}, \nu\right)+\nu W
\end{aligned}
$$

where $\mathcal{L}_{n}\left(p_{n}, \nu\right)=U_{n}\left(p_{n}\right)-\nu p_{n}$. Observing that $\mathcal{L}_{n}(\cdot)$ only depends on user $n$ 's local information, we have the dual decomposition for each user $n$. Each user solves subproblem (7) for given Lagrange multipliers $\tilde{\nu}$, i.e.,

$$
p_{n}(\tilde{\nu})=\arg \max _{p_{n}^{\min } \leq p_{n} \leq p_{n}^{\max }} \mathcal{L}_{n}\left(p_{n}, \tilde{\nu}\right), \text { for all } n .
$$


Subproblem (7) can be solved with the subgradient method, since $\mathcal{L}_{n}$ is strictly concave [27]. User $n$ iteratively updates its power $p_{n}$ until $p_{n}$ converges, as

$$
p_{n}(l+1)=\left[p_{n}(l)+\kappa(l) \cdot \partial \mathcal{L}_{n}\left(p_{n}\right) / \partial p_{n}\right]^{+}
$$

where $[\cdot]^{+}$denotes the projection of $p_{n}$ onto the range $\left[p_{n}^{\min }, p_{n}^{\max }\right]$, and $\kappa(l)$ is the step size that varies in each step $l$ according to the Armijo rule [27]. The solution $p_{n}$ can be solved locally by the users and converges to the optimal solution of $\tilde{p}_{n}$ for all $n$ as $l \rightarrow \infty$.

For a given optimal solution $\tilde{p}_{n}$, the master dual problem is to be solved by the DCC, i.e.,

$$
\begin{array}{ll}
\text { minimize }: & \mathcal{L}\left(\tilde{p}_{n}, \nu\right) \\
\text { subject to }: & \nu \geq 0, \text { for all } n .
\end{array}
$$

We can also apply the subgradient method to iteratively update the multipliers as

$$
\nu(l+1)=\max \{\nu(l)-\alpha(l) \cdot \partial \mathcal{L}(\nu) / \partial \nu, 0\}
$$

where $\alpha(l)$ is the step size. The Lagrange multipliers converges to the optimal as $l \rightarrow \infty$. Since problem (5) is a convex problem, the duality gap is zero, and the solution of (7) is unique. The primal variable $p_{n}$ will also converge to the optimal solutions [26].

The DUBMLC algorithm is presented in Algorithm 2. With DUBMLC, each user greedily maximizes its own benefit by solving (7) with current "price" $\nu$, which is controlled by the DCC through the master dual problem (9). Due to the convexity of the problem, the optimization gap is zero, and the optimal total maximum benefit is reached when the algorithm converges.

Algorithm 2: Distributed User Benefit Maximization Load Control Algorithm

1) SetAlgoLined $l=0$ and the DCC initializes nonnegative parameter $\nu(l)$;

2) The DCC announces the parameters to the users via the communications network;

3) Each user locally solves problem (7) as in (8) to obtain its requested power;

4) Each user sends its requested power to the DCC via information networks;

5) The DCC updates the parameters $\nu(l)$ as in (10) and announces the new value $\nu(l+1)$ to all users;

6) $l=l+1$ and go to Step 3, until the solution converges;

Combining GSEPS and DUBMLC, we now present the General Smooth Electric Power Scheduling Policy. Specifically, at the beginning of each period, which can be daily based or be an arbitrary period of time, the users send their slotted demand profiles to the DCC through the communications network. The users do not need to be cooperative at this step. If a user does not want to shift its load, it can set all its load as priority load.
The utility company may also set an incentive to encourage users to put more load deferrable, such as by dynamic pricing. After aggregating all the demand profiles, the DCC calculates the deterministic cumulative supply/demand curves for the power distribution networks and executes GSEPS to compute the smooth power profile. After that, the DCC let the users control their electricity usage to match the smooth schedule with DUBMLC. The load control does not necessarily need to be fully executed at the exact beginning of the period. In the second stage, the users are assumed to be cooperative since they have already selected their preference at the first stage and at least all the priority load will be granted in this step. If a user requests a usage exceeding the planned service level, the DCC may allow the distribution substation to temporally fulfill the excess demand but charging a penalty price based on the electric energy availability of the power distribution network. It may operate at some time ahead of the scheduled time slot.

\section{E. Remarks}

The proposed scheme is based on two basic assumptions: 1) prediction of the (near) future loads and 2) classification of customer loads. It is worth noting that these are the general assumptions made in many DR papers, where DR algorithms are designed to intentionally modify the consumption patterns of customers' electricity usages, such as altering the timing, the level of instantaneous demand, or the total electricity consumption. An effective DR algorithm will shift user loads from on-peak to off-peak periods depending on the consumers' preferences and lifestyles. Therefore, for DR to work, there is a need for knowing the loads and which of the loads are deferrable [6].

It is a challenging problem for users to perfectly predict their future demand in traditional power systems. However, there are also some facts that can be exploited for such prediction. For example, the electricity usage in a household usually has a daily pattern. Although there is long-term variations, the day-ahead usage is usually similar to the past-day usage. In addition, many usages are deferrable. For example, heating may be turned down, air conditioning or refrigeration may be turned up, and washer and dryer/dishwasher can work in midnight, slightly delaying the draw until a peak in usage has passed.

In the SG paradigm, with the wide deployment of smart meters and smart appliance, it is expected that load forecasting could be more accurate, particularly for short-term predictions (from $1 \mathrm{~h}$ to 1 week) [28]-[30]. The intelligent core in smart meters can track the usage history of the users and assist users to plan the service level of each smart load/appliance and send out the control commands with the service level of individual load through networks [31]. Several energy-management start-up companies, such as Retroficiency, EcoFactor, and AutoGrid, are offering effective algorithms to crunch the energy usage data so users ranging from huge factories to individual households can track and reduce waste [31].

Nevertheless, the performance of the proposed scheme depends on the difficulty or uncertainty in defining day-ahead usages by the individual customers. To deal with such uncertainties or prediction errors, some real-time generation or 
real-time electricity market will be helpful to compensate for the stochastic fluctuations in the future demand. Even in this case, the proposed scheme is still helpful since it can smooth out the bulk of the future demands and only leaves the stochastic disturbance for real-time generation to cover, thus minimizing the demand for real-time generation and still reducing the generation cost.

Note that the proposed model actually does not solely rely on the accurate load forecasting. The deterministic model is actually based on the lower and upper bounds of the user demand. Thus, some uncertainty in prediction can be accommodated. Meanwhile, due to the increasing deployment of the household energy storage system, the uncertainty of the energy may be managed by the stored energy. Even in this case, the proposed scheme is still helpful to smooth out the grid load. The integration of energy storage system (and a stochastic component in the model) into the framework could be an interesting extension for future investigation.

Another assumption in this paper is that the users are willing to be cooperative in this framework with certain incentive mechanisms (e.g., pricing). The time-of-use pricing has been implemented in many states in the U.S., and the dynamic pricing adoption is fast growing with accelerated pace [32]. Although some users are reluctant to shift their load based on the pricing strategy for now, with the rapid progress in the deployment of smart meters and smart appliances, billing software availability, two-way flow of information infrastructure, and the advances in regulation, it is reasonable to expect that the users would be more cooperative in DR in the future SG.

\section{Simulation Study}

Here, we evaluate the proposed algorithms by simulating an electric power distribution network with 250 independent users. We assume a daily period slotted into $L=144$ time slots (i.e., $\tau=10 \mathrm{~min}$ ). Clearly, the trajectories of $W_{\min }$ and $W_{\max }$ play a major role in determining the smooth power schedule. Due to the lack of real household usage data, we assume that the demand for each user during the period is randomly distributed from 35 to $50 \mathrm{kWh}$. The DCC aggregates the load profiles and generates the cumulative supply/demand curves at the beginning of the period. We adopt a benefit function $U_{n}(t)=k_{1} q_{n}(t)-(1 / 2) k_{2} q_{n}^{2}(t)$ [33], where $q_{n}(t) \in$ $[0,1]$ is the normalized value of power supply $p_{n}(t)$. With this $U_{n}(\cdot)$, problem (5) becomes a quadratic programming problem, which can be effectively solved with the proposed distributed algorithm. Without loss of generality, we set $k_{1}=k_{2}=1$ in the simulations. The default simulation settings are shown in Table II.

We first examine the performance of SEPS-DL and GSEPS. For SEPS-DL, all the electric energy demand is deferrable. For GSEPS, we assume that $50 \%$ of the demand is deferrable and that the deadlines are randomly distributed during the daily period. The cumulative demand curves and the computed schedules are plotted in Fig. 7. We find that both electric power schedules lie between $\vec{W}_{\min }$ and $\vec{W}_{\text {max }}$, which means that they are feasible and satisfying the user demands in the entire period. In some time slots, e.g., slots [70, 80] and [110, 120], GSEPS
TABLE II

Default Simulation Settings

\begin{tabular}{l|l}
\hline Parameter & Value \\
\hline Number of users & 250 \\
Simulation period & 1 day \\
Time slot duration & 10 min \\
Electric energy demand/user/day & uniformly in $[35,50] \mathrm{kWh}$ \\
Percentage of deferrable load & $50 \%$ \\
Generation cost & $g(P(t), \theta(t))=\left(\beta_{1}+\beta_{2} P(t)+\right.$ \\
& $\left.\beta_{3} P(t)^{2}\right) \theta(t)$ \\
\hline
\end{tabular}

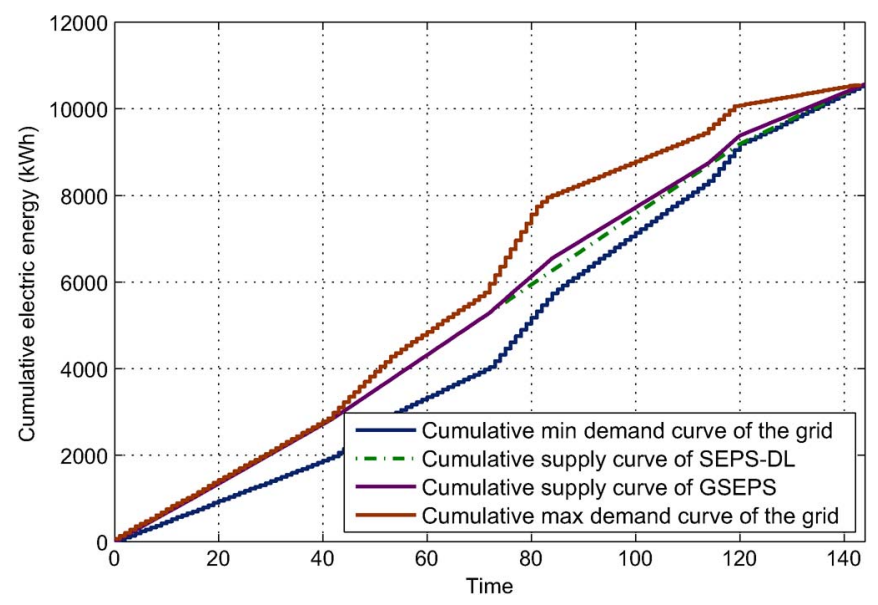

Fig. 7. Comparison of the power schedules achieved by SEPS-DL an GSEPS.

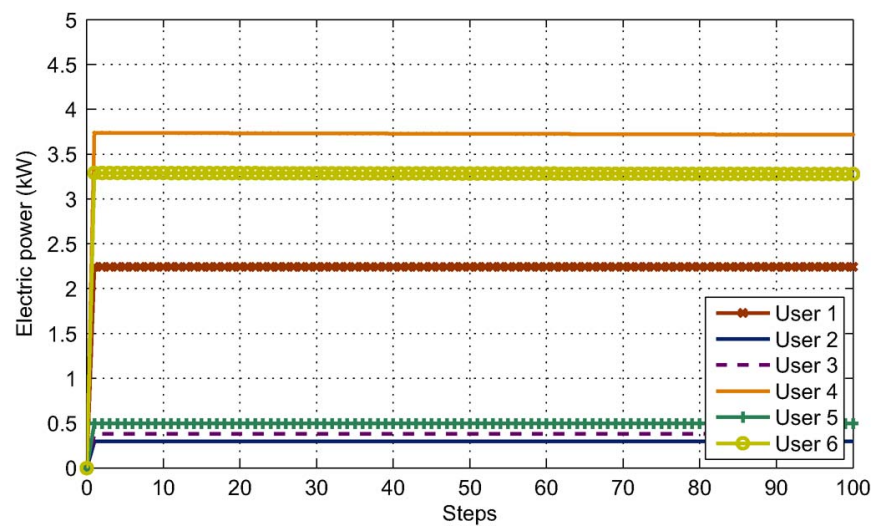

Fig. 8. Convergence of the individual power allocation achieved by DUBMLC.

requests a larger electric power than SEPS-DL. This is due to the hard requirement for the priority load, which temporally forces GSEPS to increase the electric power supply.

After the smooth power schedule is obtained, DUBMLC is executed to divide the power to individual users in each time slot. For better illustration, we only plot the power convergence curves for six users in Fig. 8. The curves for other users are similar. We find that all the curves converge to the optimal values quickly; after several steps, there is no significant variation in the electric powers of individual users. Since the proposed scheduling algorithm can be implemented as a dayahead planning strategy, the power plan for individual user can be computed in advance in practice.

We next compare the proposed algorithms with two alternatives: 1) "supply until deadline" scheme (SUDP), in which 


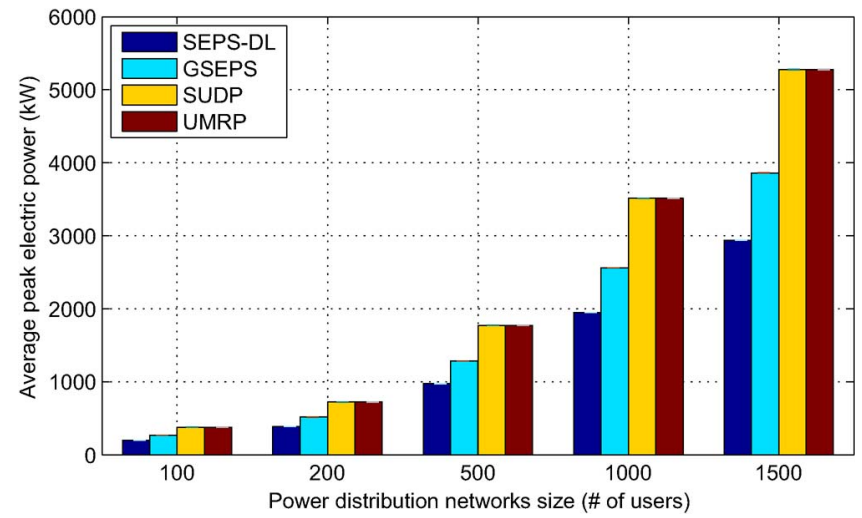

Fig. 9. Peak electric powers achieved by SEPS-DL, GSEPS, SUDP, and UMRP.

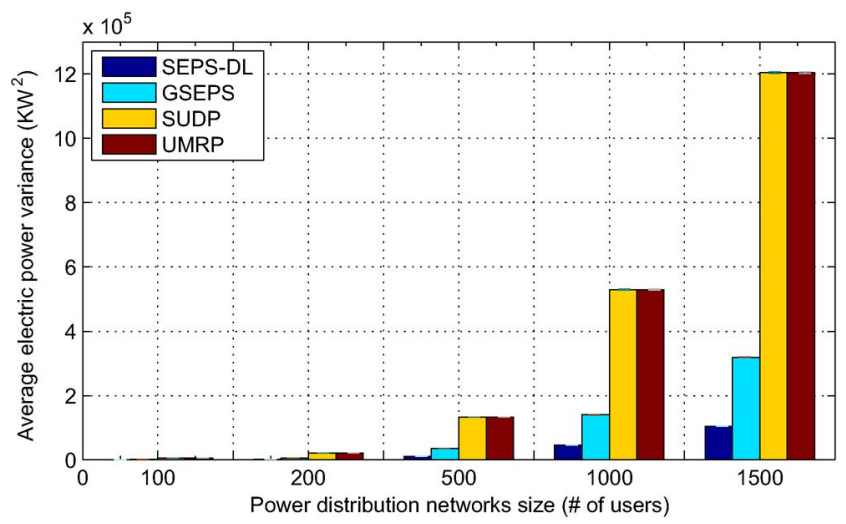

Fig. 10. Variances of the power schedules achieved by SEPS-DL, GSEPS, SUDP, and UMRP.

the deferrable load demand is served until the last minute, and 2) "utility maximization real-time pricing" scheme (UMRP), which is introduced in [34], which interestingly solves the demand-side management problem with a real-time pricing strategy and has been widely cited in the SG research community. In particular, the UMRP scheme maximizes the social welfare in an SG at each time slot independently, i.e.,

$$
\max \sum_{n \in \mathbb{U}} U_{n}\left(p_{n}(t)\right)-g(P(t), \theta(t)), \text { for all } t
$$

subject to the total power generation constraint and the user power consumption constraints. This algorithm can be extended to our simulation scenario. In this paper, the operating cost of power generation is evaluated by $g(P(t), \theta(t))=\left(\beta_{1}+\right.$ $\left.\beta_{2} P(t)+\beta_{3} P(t)^{2}\right) \theta(t)$, which is generalized from [25]. We let $\beta_{1}=120.0, \beta_{2}=6$, and $\beta_{3}=0.04$ for a generator [25] and $\theta(t)$ be uniformly distributed in $[0.5,2.5]$. This yields a quadratic programming problem, which can be solved in either a centralized or a distributed manner [34]. In the simulations, we use a centralized interior-point method to solve the problem.

The peak power and the power variation achieved by the preceding algorithms for networks with different numbers of users are presented in Figs. 9 and 10, respectively. Each number is the average of 100 simulation runs with different random seeds, with the $95 \%$ confidence interval plotted at the top of each bar. We observe that SEPS-DL and GSEPS can significantly reduce
TABLE III

Average Operating Cost of Power Generation

\begin{tabular}{l|l|l|l|l}
\hline & SEPS-DL & GSEPS & SUDP & UMRP \\
\hline \$hour & 1653 & 1669 & 1746 & 1746 \\
\hline
\end{tabular}

both peak power and power variation in the power distribution network. For example, for the network with 1000 users, SEPSDL and GSEPS achieve peak powers of 1947 and $2560 \mathrm{~kW}$, respectively, which are only $55 \%$ and $73 \%$ of the corresponding SUDP and UMRP peak powers. Similarly, we find in Fig. 10 that the SEPS-DL and GSEPS schedules are much more smoother than both the SUDP and UMRP schedules. Therefore, to design the power generation, transmission, and distribution infrastructure for this 1000-user site, we may select components, such as transformers and transmission lines, based on the capacity specifications of 1947 and $2560 \mathrm{~kW}$, respectively (with SEPS-DL and GSEPS), instead of $3513 \mathrm{~kW}$ with SUDP and UMRP. As the network size increases, the performance gap increases as well. For the smallest gap case when network size is 100 , the power reduction is $176 \mathrm{~kW}$ for SEPS-DL and $110 \mathrm{~kW}$ for GSEPS, which meets the requirement of $100-\mathrm{kW}$ minimum energy reductions for the DR products at ISONE [35].

It is interesting to observe that UMRP and SUDP have almost identical performance in the simulations. This is largely due to the choice of the object function $\sum_{n \in \mathbb{U}} U_{n}\left(p_{n}(t)\right)-$ $g(P(t), \theta(t))$. In the simulation with the preceding utility functions and cost functions, the effect of the total power decrement on the cost functions dominates the effect of the individual user power increments in their utility functions. Thus, UMRP attempts to reduce the total power generation, while only maintaining the minimum user satisfaction level. This strategy indeed degenerates UMRP to SUDP, both with similar performance. Although UMRP maximizes the welfare of the distribution network, it does not aim to smooth the power schedules. It would be helpful to carefully introduce some coefficients to balance the contributions of utility and cost to the welfare. This phenomenon also verifies our motivation of the work that the real-time pricing with utility maximization may not automatically solve the smooth electric power scheduling and peak power reduction problems. Compared with UMRP, the proposed algorithms directly target at the smoothness optimization problem and are robust to various configurations of the distribution network.

We finally compare the average generator operating costs of SEPS-DL, GSEPS, SUDP, and UMRP. Similar to the previous scenario, we assume a load site with average aggregated demand of $100 \mathrm{MW}$, which is scheduled by SEPS-DL, GSEPS, SUDP, and UMRP based on a daily period. The average generator operating costs are shown in Table III. Both SEPS-DL and GSEPS obtain smaller operating costs than SUDP and UMRP for serving the same load site. It is not surprising to see that SUDP and UMRP obtain similar results, due to the effect of the objective function. Note that UMRP is not operating cost optimal, although it seeks to maximize the social welfare. This is due to the fact that the operating cost is not independent from time slot to time slot; greedily optimizing social welfare in each time slot does not guarantee optimality over the entire period. 
Suppressing user satisfaction level some time slots may result in high power allocations at a later time slot to meet all the delayed demands right before their deadline, leading to larger peak power and electricity generation cost. On the contrary, both SEPS-DL and GSEPS optimize power scheduling over the entire period and can flexibly serve the demand to achieve smooth electric power scheduling and low operating cost.

\section{RELATED WORK}

The emergence of SG attracts new interest in evolving the next generation of power distribution systems [16]. DR is a new power distribution paradigm to reduce the peak demand and smooth demand profiles in the grid by shaping the user demands. Various implementation issues of DR in SG are examined in [35]. Real-time pricing and direct load control are two important ways to shape user demand profile.

Due to the real-time communications and control through two-way information flows in SG, new design approaches in DR are being developed recently [11]-[14], which are based on optimization and game theory approaches. In [11], the authors proposed an optimal and automatic residential energy consumption scheduling framework to achieve the tradeoff between minimization of electricity payment and appliance operation waiting time. In [12], a game theory approach is used to control the power demand at peak hours by dynamic pricing strategies. In [13], the authors studied DR for households based on utility maximization and showed that there exist time-varying prices that may achieve social optimality. A recent work [14] has introduced the framework for optimal resource allocation under the uncertainty in the two-way information network and provided a decentralized algorithm that can be implemented in practice. Although providing some interesting methods to achieve costefficient electricity usage, these papers do not explicitly address the problem of smooth electric power scheduling.

Several existing DR schemes were based on real-time pricing [7]-[10] and day-ahead load response concepts [11]-[14], aiming to maximize the social welfare or minimize the electricity payment under given demand requirements. Several recent works [4]-[6] also studied the problem of reducing the peakto-average ratio (PAR) of the electric energy consumption in SG. In [4], the authors introduced a game theory framework for a distributed algorithm to minimize the total energy payment and reduce PAR. However, users need to broadcast control messages to announce their new schedules to the entire network. The control overhead could be considerable. In [5], energy storage devices were incorporated in the SG, and user energy cost and PAR are minimized with a distributed algorithm. Although the distributed algorithm only needs to exchange information with the energy provider, the achieved Nash equilibrium cannot be guaranteed to be social optimal. In addition, this paper does not consider the operating cost of the energy provider. In a recent work [6], the authors considered a discrete time system and a time period $[0, T]$ during which $n$ electrical jobs or appliance should be scheduled, where $T$ typically is $24 \mathrm{~h}$. The problem is shown to be NP-hard, and several approximation algorithms were developed to either reduce the peak power or minimize the delay by shifting some deferrable loads.
In this paper, we consider a power distribution network with multiple users. The proposed power distribution has a twotier structure: the total demand profile for the entire network is smoothed first, and then, the scheduled power is allocated in a distributed manner to maximize their total utility. The proposed scheme is based on majorization theory. When all the load is deferrable, the proposed scheme can achieve optimal performance with respect to smoothness, peak reduction, and generation cost. Under both priority and deferrable loads, the proposed scheme also exhibits very good performance through simulations.

\section{CONCLUSION}

In this paper, we have addressed the problem of smooth electric power scheduling in a power distribution network. We have introduced a supply/demand curve model to characterize the complex relationship between electricity demand and supply. A constrained nonlinear optimization problem is formulated aiming to minimize the electric power variation and satisfy user power usage quality. We developed majorization-based algorithms for deriving smooth schedules and a distributed algorithm for allocating the power supply among users. The proposed algorithms are shown to achieve smooth power schedules, while minimizing peak power and generation cost.

\section{REFERENCES}

[1] Y. Huang, S. Mao, and R. M. Nelms, "Smooth electric power scheduling in power distribution networks," in Proc. IEEE GLOBECOMWorkshop Smart Grid Commun., Des. Perform., Anaheim, CA, Dec. 2012, pp. 1469-1473.

[2] X. Fang, S. Misra, G. Xue, and D. Yang, "Smart grid-The new and improved power grid: A survey," IEEE Commun. Surveys \& Tuts., vol. 14, no. 4, pp. 944-980, Dec. 2011.

[3] S. Shao, M. Pipattanasomporn, and S. Rahman, "Demand response as a load shaping tool in an intelligent grid with electric vehicles," IEEE Trans. Smart Grid, vol. 2, no. 4, pp. 624-631, Dec. 2011.

[4] A. Mohsenian-Rad, V. Wong, J. Jatskevich, R. Schober, and A. Leon-Garcia, "Autonomous demand-side management based on game-theoretic energy consumption scheduling for the future smart grid," IEEE Trans. Smart Grid, vol. 1, no. 3, pp. 320-331, Dec. 2010.

[5] H. K. Nguyen, J. B. Song, and Z. Han, "Demand side management to reduce peak-to-average ratio using game theory in smart grid," in Proc. IEEE INFOCOM Workshops, Orlando, FL, Mar. 2012, pp. 91-96.

[6] S. Tang, Q. Huang, X.-Y. Li, and D. Wu, "Smoothing the energy consumption: Peak demand reduction in smart grid," in Proc. IEEE INFOCOM, Turin, Italy, Apr. 2013, pp. 1133-1141.

[7] Y. Wang, S. Mao, and R. M. Nelms, "Distributed online algorithm for optimal real-time energy distribution in the smart grid," IEEE Internet Things J., vol. 1, no. 1, pp. 70-80, Feb. 2014.

[8] Y. Wang, S. Mao, and R. M. Nelms, "An online algorithm for optimal real-time energy distribution in smart grid," IEEE Trans. Emerging Topics Comput., vol. 1, no. 1, pp. 10-21, Jul. 2013.

[9] Y. Huang, S. Mao, and R. M. Nelms, "Adaptive electricity scheduling in microgrids," IEEE Trans. Smart Grid, vol. 5, no. 1, pp. 270-281, Jan. 2014.

[10] Y. Huang and S. Mao, "On quality of usage provisioning for electricity scheduling in microgrids," IEEE Syst. J., vol. 8, no. 2, pp. 619-628, Jun. 2014.

[11] A. H. Mohsenian-Rad and A. Leon-Garcia, "Optimal residential load control with price prediction in real-time electricity pricing environments," IEEE Trans. Smart Grid, vol. 1, no. 2, pp. 120-133, Sep. 2010.

[12] C. Ibars, M. Navarro, and L. Giupponi, "Distributed demand management in smart grid with a congestion game," in Proc. IEEE SmartGridComm, Gaithersburg, MD, Oct. 2010, pp. 495-500.

[13] N. Li, L. Chen, and S. H. Low, "Optimal demand response based on utility maximization in power networks," in Proc. IEEE PES Gen. Meet., Detroit, MI, Jul. 2011, pp. 1-8. 
[14] M. G. Kallitsis, G. Michailidis, and M. Devetsikiotis, "Optimal power allocation under communication network externalities," IEEE Trans. Smart Grid, vol. 3, no. 1, pp. 162-173, Mar. 2012.

[15] M. LeMay, R. Nelli, G. Gross, and C. A. Gunter, "An integrated architecture for demand response communications and control," in Proc. HICSS, Washington, DC, 2008, pp. 174-183.

[16] G. T. Heydt, "The next generation of power distribution systems," IEEE Trans. Smart Grid, vol. 1, no. 3, pp. 225-235, Dec. 2010

[17] J. Kumagai, "Virtual power plants, real power," IEEE Spectr., vol. 49, no. 3, pp. 13-14, Mar. 2012.

[18] H. Farhangi, "The path of the smart grid," IEEE Power Energy Mag., vol. 8, no. 1, pp. 18-28, Jan./Feb. 2010.

[19] J. Medina, N. Muller, and I. Roytelman, "Demand response and distribution grid operations: Opportunities and challenges," IEEE Trans. Smart Grid, vol. 1, no. 2, pp. 193-198, Sep. 2010.

[20] A. W. Marshall and I. Olkin, Inequalities: Theory of Majorization and Its Applications. New York, NY, USA: Academic, 1979.

[21] J. Salehi, Z.-L. Zhang, J. Kurose, and D. Towsley, "Supporting stored video: Reducing rate variability and end-to-end resource requirements through optimal smoothing," IEEE/ACM Trans. Netw., vol. 6, no. 4, pp. 397-410, Aug. 1998.

[22] E. Jorswieck and H. Boche, "Optimal transmission strategies and impact of correlation in multiantenna systems with different types of channel state information," IEEE Trans. Signal Process., vol. 52, no. 12, pp. 34403453, Dec. 2004.

[23] A. Papavasiliou and S. S. Oren, "Supplying renewable energy to deferrable loads: Algorithms and economic analysis," in Proc. IEEE Power \& Energy Soc. Gen. Meet., Jul. 2010, pp. 1-8.

[24] B. C. Arnold, Majorization and the Lorenz Order: A Brief Introduction. New York, NY, USA: Springer-Verlag, 1987.

[25] A. J. Wood and B. F. Wollenberg, Power Generation, Operation, and Control. New York, NY, USA: Wiley, 1984.

[26] D. P. Palomar and M. Chiang, "A tutorial on decomposition methods for network utility maximization," IEEE J. Sel. Areas Commun., vol. 24, no. 8, pp. 1439-1451, Aug. 2006.

[27] D. Bertsekas, Nonlinear Programming. Belmont, MA, USA: Athena Scientific, 1995

[28] M. Ghofrani, M. Hassanzadeh, M. Etezadi-Amoli, and M. Fadali, "Smart meter based short-term load forecasting for residential customers," in Proc. NAPS, Boston, MA, Aug. 2011, pp. 1-5.

[29] M. Chaouch, "Clustering-based improvement of nonparametric functional time series forecasting: Application to intra-day household-level load curves," IEEE Trans. Smart Grid, vol. 5, no. 1, pp. 411-419, Jan. 2014.

[30] B. Stephen, F. Isleifsson, S. Galloway, G. Burt, and H. Bindner, "Online amr domestic load profile characteristic change monitor to support ancillary demand services," IEEE Trans. Smart Grid, vol. 5, no. 2, pp. 888895, Mar. 2014

[31] B. Walsh, "Breakthrough: Smart Power-Data-crunching algorithms, not solar panels, are the new darlings of clean tech," Time Mag., p. 10, Apr. 8, 2013.

[32] Energy.gov, "Energy Policy Act of 2005," Public Law 10958, 109th Congress. [Online]. Available: http://energy.gov/eere/femp/ energy-policy-act-2005

[33] M. Fahrioglu and F. L. Alvarado, "Designing incentive compatible contracts for effective demand management," IEEE Trans. Power Syst., vol. 15 , no. 4, pp. 1255-1260, Nov. 2000.

[34] P. Samadi, A. Mohsenian-Rad, R. Schober, V. Wong, and J. Jatskevich, "Optimal real-time pricing algorithm based on utility maximization for smart grid," in IEEE SmartGridComm, Oct. 2010, pp. 415-420.

[35] F. Rahimi and A. Ipakchi, "Demand response as a market resource under the smart grid paradigm," IEEE Trans. Smart Grid, vol. 1, no. 1, pp. 8288, Jun. 2010

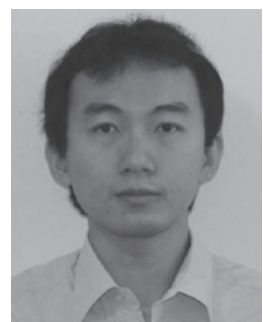

Yingsong Huang (S'12) received the B.S. degree in automation and the M.S. degrees in control theory and control engineering and from Chongqing University, Chongqing, China, and the Ph.D. degree in electrical and computer engineering from Auburn University, Auburn, AL, USA, in 2013.

From July 2003 to July 2007, he was a Senior Research and Development Engineer and a Vice-Supervisor with Advantech Corporation, Ltd., Beijing and Taipei, China. He is currently a Member of Technical Staff with NetApp, Inc., Pittsburgh, PA, USA. His research interests include modeling, control and optimization in microgrids, smart grid, and computer networks.

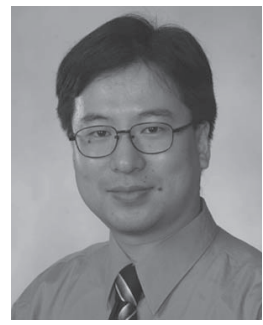

Shiwen Mao (S'99-M'04-SM'09) received the $\mathrm{Ph} . \mathrm{D}$. degree in electrical and computer engineering from Polytechnic University, Brooklyn, NY, USA.

$\mathrm{He}$ is currently the McWane Associate Professor in the Electrical and Computer Engineering Department, Auburn University, Auburn, AL, USA. His research interests include wireless networks and multimedia communications, with current focus on cognitive radio, small cells, $60-\mathrm{GHz}$ millimeterwave networks, free-space optical networks, and smart grid.

Prof. Mao is a Distinguished Lecturer of the IEEE Vehicular Technology Society in the Class of 2014. He is on the Editorial Board of the IEEE TRANSACTIONS ON WIRELESS COMMUNICATIONS, the IEEE Internet of Things Journal, and the IEEE Communications Surveys and Tutorials, among others. He was a recipient of the 2013 IEEE ComSoc MMTC Outstanding Leadership Award and the NSF CAREER Award in 2010. He was a corecipient of the IEEE ICC 2013 Best Paper Award and The 2004 IEEE Communications Society Leonard G. Abraham Prize in the field of communications systems.

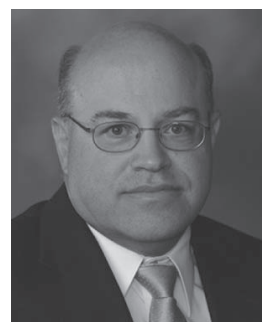

R. M. Nelms (F'04) received the B.E.E. and M.S. degrees from Auburn University, Auburn, AL, USA, in 1980 and 1982, respectively, and the Ph.D. degree from Virginia Polytechnic Institute and State University, Blacksburg, VA, USA, in 1987, all in electrical engineering.

He is currently a Professor and the Chair of the Electrical and Computer Engineering Department with Auburn University. His research interests are in power electronics, power systems, and electric machinery.

Prof. Nelms was named an IEEE Fellow "for technical leadership and contributions to applied power electronics." He is a Registered Professional Engineer in Alabama. 